\title{
Brownian dynamics simulation of a hard-sphere suspension
}

\author{
P. Strating \\ Faculty of Mathematical Sciences, University of Twente, P.O. Box 217, 7500 AE Enschede, The Netherlands
}

(Received 18 August 1998; revised manuscript received 12 October 1998)

\begin{abstract}
In this paper we discuss the nonequilibrium shear viscosity of a suspension of hard spheres that is modeled by neglecting hydrodynamic interactions in a consistent way. The aim is to establish the true capabilities of this model in predicting the properties of real suspensions. A Brownian dynamics algorithm is used to simulate the movements of hard spheres immersed in a Newtonian solvent in a nonequilibrium steady shear flow. A new development is the treatment of the overlap of spheres as elastic collisions, to simulate the no-flux boundary condition on the surfaces of rigid particles. This algorithm is compared with other algorithms suggested in the literature, and is shown to be simple and accurate even for two spheres at close distance. This provides an algorithm that is very suitable for calculating the pair distribution function and especially its hard-sphere contact value, both in equilibrium and nonequilibrium simulations. The algorithm is used to study the nonequilibrium stationary shear flow in the low shear limit. The simulations correctly reproduce the exact lowdensity limit of the perturbation of the pair distribution function. The perturbation of the pair distribution function in shear flow can be extracted from the simulation data and used to compute the stationary shear viscosity for a system of diffusing hard spheres without hydrodynamic interactions. This yields a flow curve for this model system including the low shear limit. It is found that the model shear viscosity fails at intermediate and high shear rates as can be expected from the neglect of hydrodynamic interactions, but also in the low shear limit at small and moderate volume fractions. [S1063-651X(99)14002-9]
\end{abstract}

PACS number(s): 05.70.Fh, 61.20.Ja, 51.20.+d

\section{INTRODUCTION}

Systems of hard spheres have always been the subject of extensive research. In statistical physics the hard-sphere system is studied because it is one of the simplest systems that retains many of the complexities of many-particle systems. The hard spheres are studied in the context of classical statistical physics, quantum or relativistic many-body theory, or the theory of phase transitions. In rheology the hard-sphere system often is studied as a first approximation to the properties of colloidal suspensions. Real suspensions are described with respect to their deviations from hard-sphere behavior.

In rheology the model hard-sphere system consists of hard spheres of equal size dispersed in a Newtonian solvent with viscosity $\eta_{0}$. The size of the colloidal particles is assumed to be small enough such that inertial and gravitational effects are negligible, and the velocity of the flow is such that the Reynolds number is low and turbulence is absent. This system was theoretically studied through the pioneering work of Batchelor [1,2], while important measurements were done by Van der Werff and De Kruif [3], with viscosity measurements dispersions of silica spheres in cyclohexane at various particle sizes and concentrations, and Ackerson and Pusey [4], who studied the nonequilibrium structure of suspensions in shear flow. In theoretical work this model system is rigorously described by the $N$-particle hard-sphere diffusion equation, which includes the diffusion and hydrodynamic interactions of hard spheres, and possibly an external flow field. Proper understanding of the hard-sphere suspension is indispensable if a comparison with a real-life suspension is to yield additional information, which explains the continuous theoretical work on hard-sphere suspensions.

The main difficulty with the statistical theory of suspen- sions is that the hydrodynamic interactions of the colloidal particles are not pairwise additive as is the case in classical theories of molecular fluids. In suspensions, a change in position or velocity of a suspended particle instantaneously influences the entire flow field and the velocities of all other particles. One approach is to use series expansions in the particle number density to obtain results at higher densities, but this has not been a successful approach, because the computations become extremely cumbersome even at moderate volume fractions. A different approach is to restore the connection with classical statistical physics, and to model the suspension by neglecting the hydrodynamic interactions, but at the same time, to take into account the pairwise hardsphere interactions rigorously. The basic idea of this approach is that at high densities the hard-sphere interactions may become dominant, and that the properties of the suspension are determined largely by the hard-sphere packing structures. This idea has been conjectured by several authors [5-9], and has been applied, in various forms, fairly successfully in the low shear regime.

If hydrodynamic interactions are neglected, an exact expression for the shear viscosity of a hard-sphere suspension can be given in terms of the nonequilibrium pair distribution function $[10-12,9]$. This will be discussed in Sec. II. The main theoretical challenge then reduces to computing the pair distribution function in shear flow. In this paper we will take the route of computing the nonequilibrium pair distribution function from Brownian dynamics simulations that are equivalent to the basic hard-sphere suspension model. In this way it is possible to rate the modeling of a hard-sphere colloidal suspension as a collection of diffusing hard spheres, without blurring the comparison with series expansion truncations or equation three body closures $[13,14]$.

In recent years there seems to be a growing unanimity that 
suspension models without hydrodynamic interactions cannot yield correct results. An important reason is that shear thickening behavior has been found in experiments $[15,16]$ at high shear rates, and in simulations of suspensions with hydrodynamic interactions $[17,18]$. This shear thickening behavior seems to be rooted in hydrodynamics, because it has not been found in simulations of models lacking hydrodynamic interactions so far, and because a possible hydrodynamic mechanism for shear thickening at high shear rates was conjectured in [19].

However, before the model suspension without hydrodynamic interactions is discarded as a model for real suspensions, it should be tested thoroughly. The aim of this paper, and a previous paper on the stress tensor [12], is to treat the model suspension without hydrodynamic interactions in a completely consistent way without additional approximations or corrections. In our opinion only such a procedure may establish definitely whether or not the model falls short in describing real suspensions.

The new aspects in this paper are the following. First, in Sec. II, an exact expression for the shear viscosity in terms of the pair distribution function is given and shown to be valid at all shear rates. Because the expression for the shear viscosity requires the calculation of the nonequilibrium pair distribution function, we will develop a new method to deal with hard-sphere overlaps in Brownian dynamics simulations in Sec. III. This method is discussed and compared to two other simulation algorithms, and it is concluded that the new algorithm is the most appropriate algorithm. In Sec. IV we discuss the application of the formalism to nonequilibrium shear flow simulations, especially in the low shear regime. The shear viscosities obtained from the simulations are compared with experimental work on real suspensions.

\section{STEADY-STATE SHEAR VISCOSITY}

In this paper we aim at calculating the relative zero shear viscosity for a hard-sphere suspension which is modeled by neglecting the hydrodynamic interactions in a consistent way. Although hydrodynamic interactions are neglected, the solvent still plays an important role, because it corroborates the diffusion of the particles and supplies friction that drags the colloidal particles along with the flow. This is sometimes called the free-draining model of suspensions [5]. The time evolution for this model is described by the $N$-particle Smoluchowski equation [20]

$$
\frac{\partial}{\partial t} P^{N}(t)=-\sum_{i=1}^{N} \frac{\partial}{\partial \mathbf{r}_{i}} \cdot\left[\mathbf{u}_{i} P^{N}-D_{0} \frac{\partial}{\partial \mathbf{r}_{i}} P^{N}+\frac{\mathbf{F}_{i}}{\zeta_{0}} P^{N}\right] .
$$

In this equation we have introduced the $N$-particle distribution function $P^{N}\left(\mathbf{r}_{1} \cdots \mathbf{r}_{N} ; t\right)$, the Stokes' friction coefficient $\zeta_{0}$, and $D_{0}$, the "free" diffusion coefficient of a single particle in the solvent given by $D_{0}=k_{B} T / \zeta_{0}\left(k_{B}\right.$ is the Boltzmann constant, $T$ is the temperature); $\mathbf{u}_{i}$ is the (local) flow velocity at the position of particle $i$, and $\mathbf{F}_{i}$ is the total force on particle $i$. From a macroscopic point of view, i.e., when the colloidal particles are considered to be microscopic, this evolution equation has a role similar to the role of the Liouville equation in statistical mechanics.
The macroscopic shear viscosity for this model suspension can be defined as the ratio of the $x y$ components of the stress tensor and the shear rate. Well-known expressions for the stress tensor in colloidal suspensions of spherical particles are given by Batchelor [2,1] and Brady [11]. In Refs. [12] and [21] the relations between the different contributions in the stress tensor expressions of Batchelor and Brady were demonstrated.

In our model suspension of identical hard spheres, the solvent gives a contribution $\eta_{0}$ to the viscosity. If inertial effects are neglected, there are no kinetic contributions to the stress tensor and therefore to the viscosity. Also, if hydrodynamic interactions are omitted, single-particle hydrodynamics remains and the stresslet contribution takes on its singleparticle form at all densities and shear rates. This gives the Einstein contribution to the viscosity $[22,23]$ according to a calculation as in Landau and Lifshitz [24]. The remaining part of the stress tensor can be considered either as an interaction or "Brownian" contribution, as discussed in [12].

So, defining the shear viscosity $\eta$ as the ratio of the $x y$ components of the stress tensor and shear rate $\dot{\gamma}$, we obtain for this model suspension the following expression for the steady-state shear viscosity

$$
\eta=\eta_{0}+\frac{5}{2} \varphi \eta_{0}-\frac{1}{2} \frac{k_{B} T n^{2}}{\dot{\gamma}} \int \frac{x y}{r} \delta(r-\sigma) g(\mathbf{r}) d \mathbf{r} .
$$

In this expression, which is similar to expressions of, e.g., Brady [11] and De Schepper [10], $n$ is the overall particle number density and $g(\mathbf{r})$ is the pair distribution function. The hard-sphere diameter $\sigma$ will be used as the length scale in this paper, so we set $\sigma=1$. The pair distribution function is a well-known reduced form [25] of the $\mathrm{N}$-particle distribution function $P^{N}$ in Eq. (2.1). The $\delta$ function in the integrand reflects the nondifferentiable character of the interparticle potential. It is emphasized that this expression is exact for this model of a suspension of hard spheres, because all possible contributions to the stress tensor are considered. It is possible to maintain [5] that in Eq. (2.2) the hydrodynamic contribution $\eta_{0}+\frac{5}{2} \varphi \eta_{0}$ may be replaced by $\eta_{\infty}$, the viscosity at infinite steady shear rate (e.g., as obtained from the Krieger-Dougherty formula [26]), thus improving the model's results in certain regimes. This however, it is not consistent with the model of single-particle hydrodynamics, and beclouds the rating of the present model without hydrodynamic interactions as a model for real suspensions.

The task remaining is to calculate the nonequilibrium pair distribution function $g(\mathbf{r})$. This task is complicated because in shear flow $g(\mathbf{r})$ is both a function of the number density and the shear rate: the normal equilibrium pair distribution (as obtained, e.g., from the Percus-Yevick equation) gives no contribution to the viscosity due to its spherical symmetry. A theoretical approach is to derive an equation for $g(\mathbf{r})$ from Eq. (2.1). The resulting equation, however, involves unknown higher order distribution functions, requiring equation closures $[13,9]$. In this paper we will take a different path by using computer simulations to compute $g(\mathbf{r})$. But first we will discuss some general properties of the pair distribution function in the case of shear flow. 


\section{A. Low shear limit: Perturbation expansion of $g$}

In the limit of low shear rates, it can be shown [27] that the pair distribution function, being a function only of $\mathbf{r}$, the volume fraction $\varphi$ and the shear rate (of which a dimensionless measure is the Péclet number $\mathrm{Pe}=\frac{1}{4} \dot{\gamma} \sigma^{2} / D_{0}$, see also Sec. III A), up to first order must have the form

$$
g(\mathbf{r})=g_{0}(r)+2 \mathrm{Pe} \frac{x y}{r^{2}} g_{1}(r)+O\left(\mathrm{Pe}^{2}\right),
$$

with $r=|\mathbf{r}|$, and assuming a regular series expansion of $g$ in the shear rate.

Only the perturbation of $g(\mathbf{r})$ gives a nonzero contribution to the shear viscosity. Performing the angular integrations in Eq. (2.2) we find for the relative zero shear viscosity

$$
\frac{\eta}{\eta_{0}}=1+\frac{5}{2} \varphi-\frac{36}{5} \varphi^{2} g_{1}(1)
$$

In this expression $g_{1}(1)$ is the contact value of the first-order perturbation of the pair distribution function. In order to reproduce the experimental results we necessarily need a negative contact value for $g_{1}(1)$ with an increasing magnitude for increasing volume fractions. The zero number density limit of $g_{1}(1)$ was calculated by Batchelor [28] from the twoparticle Smoluchowski equation. An equation for the lowdensity pair distribution function $g(\mathbf{r})$ can be obtained from Eq. (2.1) by using $\mathbf{u}(\mathbf{r})=\dot{\gamma} y \mathbf{e}_{x}$, integrating over all particle coordinates but two, and discarding all terms of higher order in the number density $n$. This yields the equation

$$
\nabla \cdot\left(\dot{\gamma} y \mathbf{e}_{x} g-2 D_{0} \nabla g\right)=0
$$

with the boundary condition $\hat{\mathbf{r}} \cdot\left[2 D_{0} \boldsymbol{\nabla} g-\dot{\gamma} y \mathbf{e}_{x} g\right]=0$ for $r$ $=1$. In a weak flow expansion, the pair distribution function will have the form of Eq. (2.3). With this ansatz, the twoparticle Smoluchowski equation simplifies to

$$
g_{1}^{\prime \prime}+\frac{2}{r} g_{1}^{\prime}-\frac{6}{r^{2}} g_{1}=0
$$

and $g_{1}^{\prime}(1)=1$. The solution of this equation is

$$
g_{1}(r)=-\frac{1}{3 r^{3}},
$$

which predicts a nonequilibrium low shear, low-density contact value of $-\frac{1}{3}$. This result provides an important check on the nonequilibrium simulations.

\section{B. High shear rates: Expansion in spherical harmonics}

A more general approach to Eq. (2.2) is the following. At all shear rates, $g(\mathbf{r})$ can be expanded in spherical harmonics [29] as

$$
g(\mathbf{r})=\sum_{l=0}^{\infty} \sum_{m=-l}^{l} G_{l m}(r) Y_{l m}(\theta, \phi) .
$$

The (complex) $Y_{l m}(\phi, \theta)$ are orthogonal on a sphere

$$
\int Y_{l^{\prime} m^{\prime}}^{*} Y_{l m} d \Omega=\delta_{l^{\prime} l} \delta_{m^{\prime} m}
$$

( $\Omega$ denoting the angular variables), and satisfy $Y_{l,-m}$ $=(-1)^{m} Y_{l m}^{*}$. With the identity

$$
\begin{aligned}
\frac{x y}{r^{2}} & =\cos \phi \sin \phi \sin ^{2} \theta=\frac{1}{i} \sqrt{\frac{2 \pi}{15}}\left(Y_{22}-Y_{2,-2}\right) \\
& =\frac{1}{i} \sqrt{\frac{2 \pi}{15}}\left(Y_{2,-2}^{*}-Y_{22}^{*}\right)
\end{aligned}
$$

and the orthogonality, we can rewrite the angular integral in Eq. (2.2),

$$
\begin{aligned}
& \int \frac{x y}{r^{2}} g(\mathbf{r}) d \Omega \\
&=\int \frac{1}{i} \sqrt{\frac{2 \pi}{15}}\left(Y_{2,-2}^{*}-Y_{22}^{*}\right) \\
& \quad \times \sum_{l=0}^{\infty} \sum_{m=-l}^{l} G_{l m}(r) Y_{l m}(\theta, \phi) d \Omega \\
&=\frac{1}{i} \sqrt{\frac{2 \pi}{15}} \int\left(Y_{2,-2}^{*}-Y_{22}^{*}\right)\left[G_{2,-2} Y_{2,-2}+G_{22} Y_{22}\right] d \Omega \\
&=\frac{1}{i} \sqrt{\frac{2 \pi}{15}} \int\left(Y_{2,-2}^{*}-Y_{22}^{*}\right)\left[\frac{1}{2}\left(G_{2,-2}+G_{22}\right)\left(Y_{2,-2}+Y_{22}\right)\right. \\
&\left.+\frac{1}{2}\left(G_{2,-2}-G_{22}\right)\left(Y_{2,-2}-Y_{22}\right)\right] d \Omega \\
&= \frac{1}{i} \sqrt{\frac{2 \pi}{15}} \int\left(Y_{2,-2}^{*}-Y_{22}^{*}\right) \\
& \times\left[\frac{1}{2}\left(G_{2,-2}-G_{22}\right)\left(Y_{2,-2}-Y_{22}\right)\right] d \Omega .
\end{aligned}
$$

The last line shows that the viscosity in our model, i.e., if hydrodynamic interactions are neglected, is only dependent on the $r$, Pe-dependent amplitude of the part of the pair distribution function that has the functional form $Y_{2,2}-Y_{2,-2}$ $\sim \cos \phi \sin \phi \sin ^{2} \theta$. This combination was assumed in the low shear expansion in Eq. (2.3), but we have now shown that the calculation for the shear viscosity through a specific component of the nonequilibrium pair distribution function is valid for all shear rates.

In the remainder of this paper we will define $g_{1}$ $=g_{1}(r, \mathrm{Pe})$ as the amplitude function of the functional form $\cos \phi \sin \phi \sin ^{2} \theta$, without reference to the low shear expansion. As a result we can write the relative shear viscosity in the simple form

$$
\frac{\eta(\mathrm{Pe})}{\eta_{0}}=1+\frac{5}{2} \varphi-\frac{18}{5} \varphi^{2} \frac{g_{1}(1, \mathrm{Pe})}{\mathrm{Pe}}
$$

valid for this suspension model at all shear rates.

\section{SIMULATION ALGORITHM}

In computer simulations of many particle systems, the movements of the individual particles are followed in time. 
In molecular dynamics the Newton equations of motion are integrated in time, as is appropriate for gases and atomic liquids. Well known are the simulations of the hard-sphere gas by Alder and Wainwright [30]. When applied to suspensions, both colloidal particles and solvent particles have to be simulated, leading to unacceptably long computation times. Recently, interesting results for suspensions have been reported by Koelman and Hoogerbrugge [31] using solvent particles with simplified dynamics. In Brownian dynamics the Langevin equation is simulated, which describes colloidal particles that are subject to small random forces, which model the interaction with the solvent. Thus the solvent is eliminated from the simulation, reducing the number of degrees of freedom enormously. If the solvent friction is high and the velocities of the (colloidal) particles are strongly damped, one can simplify the dynamics further, assuming instantaneous equilibrium in momentum space and considering only the differential equations for the positions. The motion of a particle is then described with a Langevin-type equation in which a random displacement term generates the diffusion process. This Brownian dynamics simulation algorithm originates from the work of Ermak and McCammon [32]. If hydrodynamic interactions are included through the Oseen tensor, the simulation is referred to as a Stokesian dynamics simulation, mainly developed by Bossis and Brady [33], and applied to monolayers of spheres. Only recently this method was applied to three-dimensional colloidal suspensions in the work of Phung [17]. A completely different approach is to solve the Stokes flow completely with a boundary integral equation method, as in the work of Toose [34]. However, inclusion of Brownian motion (in the sense of thermal movement) within this framework is not straightforward, and the simulations are extremely costly regarding computation time when many particles are involved, especially in three spatial dimensions.

As mentioned earlier in the Introduction, we are interested in the properties of the system described by the $\mathrm{N}$-particle Smoluchowski equation (2.1) without hydrodynamic interactions. This equation describes $N$ freely diffusing particles within a volume $V$. The diffusion of a particle is only limited by the boundaries as set by the other $N-1$ particles. The $N$-particle Smoluchowski equation is a Fokker-Planck equation for the distribution function of the stochastic position variables. It has been known for some time that for every Fokker-Planck equation there is a corresponding stochastic differential equation (Langevin equation) with appropriate stochastic properties, such that the generated distribution functions are equivalent to the solutions of the FokkerPlanck equation [35]. The task is to find the Langevin-type equation that is equivalent to the $\mathrm{N}$-particle Smoluchowski equation. The pair distribution function may be extracted from such a simulation, rather than calculated analytically. The advantage of the simulation is that the results are in a sense exact for the model (apart from numerical or statistical error, or finite simulation size effects), while analytical methods involve approximations such as truncations of infinite series, or introduction of equation closures.

The Brownian dynamics method described below shows much resemblance with a Monte Carlo simulation to determine the configurational partition function [25]. In this paper, however, it is a dynamic simulation, because the random displacements that are carried out in Monte Carlo simulations, are not just invented to sample the configuration space, but simulate the actual diffusion of particles in time. Another difference is that an acceptance criterion is missing in our simulation, as we will see later. The simulations described show close resemblance to the work described by Heyes in a series of papers [5,36,37], but in these papers the viscosity is computed from the simulations in a different way.

For hard-sphere gases, molecular dynamics simulations are numerous. In hard sphere gases the trajectories including successive collisions of particles can be calculated exactly. An advantage of Brownian dynamics over molecular dynamics is that the simulation (and theoretical) problem of the heating up of the system in nonequilibrium simulations does not occur, because the velocities of the colloidal particles have been eliminated by assuming equilibrium in the momentum variables. A disadvantage of the elimination of velocities is that there is no obvious way of dealing with the hard sphere interactions anymore. To avoid this problem, most Brownian dynamics simulations (without hydrodynamic interactions) use differentiable potentials, such as the Lennard-Jones potential [38-40]. Although the calculation of the interparticle forces is time consuming, the implementation in the simulation algorithm is straightforward. The hard-sphere suspension without hydrodynamic interaction was recently considered by Cichocki and Hinsen [41] and Schaertl and Sillescu [42]. Their (equilibrium) simulation algorithms will be discussed shortly. These programs were developed to establish self-diffusion coefficients and phase transitions. In this paper we will see that the treatment of particle overlaps in these programs is inappropriate for nonequilibrium simulations of diffusing hard spheres in shear flow, and we will present an elegant alternative.

\section{A. Diffusion}

The method of Brownian dynamics is widespread and we will only discuss the basic equations. In a finite box $N$ particles with diameter $\sigma$ are followed in time. First important parameter is the volume fraction $\varphi$ related to the particle number density $n$ as $\frac{1}{6} \pi \sigma^{3} n$.

Diffusion is simulated by small random displacements at each time step, based on a Langevin-like equation,

$$
\dot{\mathbf{r}}_{i}=\mathbf{u}\left(\mathbf{r}_{i}\right)+\frac{\mathbf{F}_{i}}{\zeta_{0}}+\Delta \mathbf{r}_{i}(t),
$$

where $\mathbf{u}$ is the local solvent velocity, $\zeta_{0}$ is the Stokes friction coefficient (related to the solvent viscosity $\eta_{0}$ as $\zeta_{0}$ $\left.=3 \pi \eta_{0} \sigma\right), \mathbf{F}_{i}$ the force on the particle due to other particles or an external field, and $\Delta \mathbf{r}_{i}(t)$ are small random displacements with zero mean. Space is scaled with the particle diameter $\sigma$, and we introduce as unit of time the structural relaxation time

$$
\tau_{\sigma}=\frac{\sigma^{2}}{D_{0}},
$$

which sets the diffusion time scale. The dimensionless time $\tau$ is defined as $\tau=t / \tau_{\sigma}$. 
After time $\Delta \tau$ the probability for a step $\Delta \mathbf{r}$ is a Gaussian distribution function with root mean square $\delta$ $=\sqrt{2 D_{0} \Delta t / \sigma^{2}}=\sqrt{2 \Delta \tau}[35]$ :

$$
\begin{aligned}
P(\Delta \mathbf{r})= & \frac{1}{(4 \pi \Delta \tau)^{3 / 2}} \exp \left(-\frac{1}{2}\left[\left(\Delta x^{2} / 2 \Delta \tau\right)+\left(\Delta y^{2} / 2 \Delta \tau\right)\right.\right. \\
& \left.\left.+\left(\Delta z^{2} / 2 \Delta \tau\right)\right]\right)
\end{aligned}
$$

in accordance with the Einstein relation

$$
|\Delta \mathbf{r}|^{2}=6 D_{0} \Delta t=6 \sigma^{2} \Delta \tau
$$

The diffusion is superposed on a shear flow with shear rate $\dot{\gamma}$. In dimensionless units

$$
\widetilde{\mathbf{u}}\left(\tilde{\mathbf{r}}_{i}\right)=\mathbf{u}\left(\mathbf{r}_{i}\right) \frac{\tau_{\sigma}}{\sigma}=\dot{\gamma} y_{i} \mathbf{e}_{x} \frac{\tau_{\sigma}}{\sigma}=4 \text { Pe } \tilde{y}_{i} \mathbf{e}_{x},
$$

with $\mathbf{e}_{x}$ the unit vector in the $x$ direction. The dimensionless Péclet number Pe in this paper is defined as

$$
\mathrm{Pe}=\frac{1}{4} \frac{\dot{\gamma} \sigma^{2}}{D_{0}},
$$

and measures the relative motion of two particles due to shear flow as compared to relative motion due to diffusion. The factor $\frac{1}{4}$ is added to conform to the usual definition Pe $=\dot{\gamma} a^{2} / D_{0} \quad\left(a=\frac{1}{2} \sigma\right.$ is the particle radius $)$.

To reduce the effects of the finite simulation size, periodic boundary conditions are applied: the particles are supplemented with periodic images in all spatial directions. In the case of shear flow we use the Lees-Edwards construction [43]. This means that the image boxes move with the shear flow according to their positions.

\section{B. Particle overlaps or collisions}

In one time step all particles are moved at the same time with a random displacement (and in nonequilibrium simulations a displacement due to shear flow). After the displacement particles may overlap, and the displacements of these particles somehow have to be adjusted. We discuss three approaches, one by Cichocki and Hinsen [41], one by Schaertl and Sillescu [42], and a new approach.

\section{Cichocki and Hinsen method}

In the Cichocki and Hinsen simulation in each step only one particle is (Gaussian) displaced in time $\Delta \tau / N$. If an overlap occurs, the particle is restored to its previous position, which is exactly the procedure of Monte Carlo simulations. In nonequilibrium simulations, for which displacement due to shear flow may occur, this algorithm poses some problems. This can be seen as follows. Suppose all Brownian particles in row in one time step perform an illegal, overlap move, and are kept at their original position. After one time step, all particles are at the same position, but the image boxes have moved. This may cause overlap with image particles even with fixed particle positions. Although this seems a rare situation, it was found in practice that problems with the time marching scheme occurred even at moderate number densities.

\section{Schaertl and Sillescu method}

In the Schaertl and Sillescu simulations all particles are displaced simultaneously. The displacements are always of the same magnitude and the direction is only one of six Cartesian directions. After a number of steps this procedure has diffusion statistics. If an overlap has occurred between two particles, these two particles are separated to contact in the direction of their relative vector (this algorithm was also proposed in [5]). A second correction must be done if particles still overlap after the first correction step, either because there was a three particle overlap in the first place, or because a secondary overlap was generated by the correction. Such secondary overlaps occur easily, especially at high volume fractions. Schaertl and Sillescu ignore secondary overlap, because its correction is time consuming, and they are mainly interested in a very fast algorithm. Large errors are made at high volume fractions and at long simulation times, that must be corrected through the introduction of effective volume fractions.

We feel that allowing overlaps is not the way to deal with the subtleties of hard-sphere systems in an age of unrivaled computing power, and we modify the Schaertl and Sillescu algorithm the following way. First, the displacements are sampled from a Gaussian to simulate diffusion statistics from the start. Second, secondary overlaps are treated similarly as primary overlaps, i.e., the algorithm is repeated. Obviously, the algorithm is slowed down by these adjustments. However, we find that the number of repetitions of the overlap correcting loops is typically between 1 and 4 at volume fractions up to 0.55 , provided the displacement step is not chosen too large. Moreover, it is found that a nonoverlapping state is always corroborated, and there is no need to introduce effective volume fractions to correct the results. In this paper we will refer to this modified Schaertl and Sillescu algorithm still as the Schaertl and Sillescu algorithm.

\section{Elastic collision method}

In this paragraph we discuss a novel method based on the following observation. The random displacement of a colloidal particle away from any boundary is sampled from a Gaussian as discussed above. Close to a restricting wall, however, the random displacement should be sampled from a distribution function that is the solution of the Smoluchowski equation near a boundary, i.e., with a no-flux boundary condition, instead of a "free"' Gaussian. Therefore we need a method that generates the no-flux boundary condition. In our simulation we use elastic binary collisions to adjust the positions after an overlap detection, because the no-flux boundary condition eventually is the result of elastic collisions. As each particle has moved by an amount $\Delta \mathbf{r}_{i}$ in a time step $\Delta \tau$ it can be ascribed a "velocity" $\mathbf{v}_{i}=\Delta \mathbf{r}_{i} / \Delta \tau$. The procedure is now straightforward to calculate the corrected displacements with two-particle collision dynamics. The elastic collision laws prescribe conservation of energy, momentum, and angular momentum, the combination of which yields the binary collision laws 


$$
\begin{aligned}
& \mathbf{v}_{1}^{*}=\mathbf{v}_{1}+\left(\hat{\mathbf{r}}_{21} \cdot \mathbf{v}_{21}\right) \hat{\mathbf{r}}_{21}, \\
& \mathbf{v}_{2}^{*}=\mathbf{v}_{2}-\left(\hat{\mathbf{r}}_{21} \cdot \mathbf{v}_{21}\right) \hat{\mathbf{r}}_{21} .
\end{aligned}
$$

These relations describe the instantaneous velocity of two particles just after collision in terms of precollision quantities. The vector $\hat{\mathbf{r}}_{21}$ is the unit relative position vector at the moment of collision and $\mathbf{v}_{21}$ the relative velocity before the collision. Implementation of these collision laws in a Brownian dynamics algorithm will conserve the center of mass displacement and rotational displacement, which may be very important in the case of shear flow, where a net displacement or rotation is very probable. Moreover, this algorithm is consistent with the derivation of the Brownian contribution to the stress tensor in Eq. (2.2).

There are two methods to implement the elastic collision method. One method is to disregard any order in the occurrence of collisions in one time step. With this implementation all particles are displaced simultaneously, after which overlapping pairs are simply sought and corrected in the order of detection. This yields a simple and efficient algorithm. A second method is to take into account the order of collisions more or less rigorously. In that case all collision times for overlapping pairs have to be computed and ordered. A disadvantage is that after the first collision/overlap correction the list of collision times has to be recomputed. In spite of its disadvantages it was necessary to implement the second method rigorously, because it was found that only the rigorous method produced the correct close contact statistics, as described in the next section.

\section{Equilibrium comparison of the algorithms}

In Fig. 1(a) we plotted the equilibrium pair distribution function in simulations with the three different overlap correction algorithms, with 512 particles at a volume fraction of $\varphi=0.45$, and a mean squared step of $\delta=0.01 \sigma$. If we compare the results of these three algorithms, it is seen that the Schaertl and Sillescu algorithm strongly increases the possibility to find two particles at contact, resulting from putting pairs at contact after each overlap. This increased contact value decreases the possibility to find pairs at different relative distances. The Cichocki and Hinsen method and the elastic collision method produce the same pair distribution function. The difference can be seen better from a closeup of the contact region, shown in Fig. 1(b). While from Fig. 1(a) the difference seems small, it is now observed that for the Schaertl and Sillescu method, even disregarding the contact value point which has an absurd value, an estimation of the contact value based on the remaining points will be off by almost $10 \%$. It is noted that only the rigorous elastic collision method produces the same close contact statistics as the Cichocki and Hinsen method. Every different collision mechanism, or order change of collisions, influences more or less the shape of pair distribution function in the contact region. This has been checked with various (erroneous) collision mechanisms and collision orders.

Because of the importance of the hard sphere contact value in the second part of this paper, we conclude that the rigorous elastic collision algorithm is the most appropriate algorithm for our purposes. The algorithm proposed by
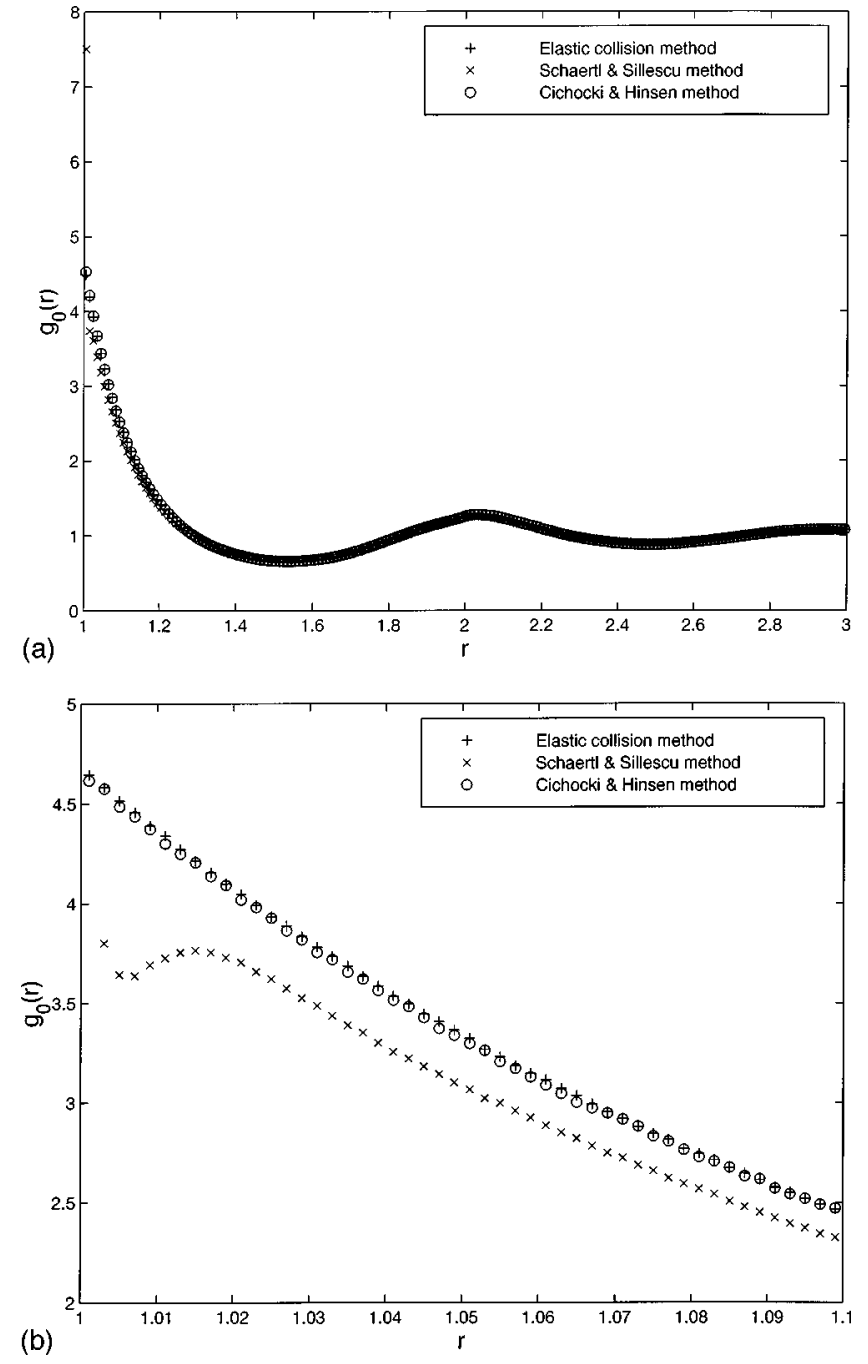

FIG. 1. Comparison of the equilibrium pair distribution function from computer simulations with three different particle overlap correction algorithms. The simulation size is 512 particles at a volume fraction of $\varphi=0.45$. (a) Global view and (b) closeup in the contact value region. Note the high contact value (between 7 and 8 ) of the Schaertl-Sillescu method in (a).

Cichocki and Hinsen [41] cannot be extended to nonequilibrium simulation without an additional overlap correction method, while the algorithm of Schaertl and Sillescu [42] is inferior in the region of interest, knowing that even at moderate volume fractions the pair distribution function varies rapidly in the region close to contact. Moreover, it can be expected that deficiencies in the overlap correction method will have a larger effect on the results in nonequilibrium simulations, because in nonequilibrium simulations the average net rotation or displacement of a pair of particles is nonzero.

With respect to the speed or computational efficiency of the algorithm we can say the following. First we find that all algorithms are comparable in speed. Most important limitation of the simulations is that the number of pairs is quadratic in the number of particles. This, however, can be largely resolved by keeping track of neighboring particles in "neighbor lists" in the familiar way [44]. If the rigorous elastic collision is implemented with neighbor lists and lists of collision times that are updated carefully, a very fast simu- 
lation code results that is easily capable of simulating several thousands of particles on a modern work station.

Summarizing we say that the rigorous elastic collision algorithm seems most appropriate for our purposes in comparison with the algorithms proposed by Cichocki and Hinsen [41] and Schaertl and Sillescu [42]. The elastic collision method makes an accurate and fast simulation of Brownian hard spheres possible. No overlaps ever occur, even at high densities, which is a serious problem in the simulations of Schaertl and Sillescu. Also, overlap-related infinite loops do not occur in the simulations. Only with the elastic collision method are collisions taken into account accurately with conservation of net displacement and rotation, which may be crucial in nonequilibrium simulations. As compared to hardsphere gas simulations, the only deficiency is that particles may pass parallel without sensing each other. For small time steps however, this is a rare event.

\section{COMPUTER SIMULATIONS}

\section{A. Calculation of the pair distribution function from finite box simulations}

The pair distribution function can be found as follows [44]. The number of particles found in a volume $d \mathbf{r}$ at distance $\mathbf{r}$ from a specified particle is $n g(\mathbf{r}) d \mathbf{r}$. The number of particles in a spherical shell of width $d r$ is

$$
d N_{r}=n g(r) 4 \pi r^{2} d r .
$$

Counting all pairs in the simulation box gives (twice) $g(r)$. In a finite box this is not completely correct. Because the box contains $N$ particles, the number of particles that can be found elsewhere in the box when one particle has been fixed in the origin, is $N-1$. To obtain a pair distribution function that approaches 1 at large separation or low volume fractions we should normalize $g$ with $N-1$ instead of $N$. This correction is negligible for large numbers of particles.

The perturbation of the pair distribution function is less easily found due to statistical error for two reasons. First, the perturbation is small by itself at low shear rates, because it is proportional to the shear rate, and second, the perturbation is not only a function of $r$ but of $\mathbf{r}$. However, the latter objection can be avoided, because in Sec. II B we showed that the viscosity in our model is determined completely by the amplitude of a combination of spherical harmonics proportional to $\cos \phi \sin \phi \sin ^{2} \theta$. The amplitude function $g_{1}(r)$ can be found from $g(\mathbf{r})$ by multiplying with $\cos \phi \sin \phi \sin ^{2} \theta$ $\left(=x y / r^{2}\right)$ and integrating over all angles $\phi$ and $\theta$. Thus, if $g$ is written

$$
g(\mathbf{r})=g_{0}(r)+\frac{x y}{r^{2}} g_{1}(r),
$$

the number of particles in a volume $d \mathbf{r}$ is

$$
d N_{r, \phi, \theta}=n\left(g_{0}(r)+\frac{x y}{r^{2}} g_{1}(r)\right) r^{2} \sin \theta d r d \phi d \theta .
$$

After multiplying with $\cos \phi \sin \phi \sin ^{2} \theta$ and integration over the angles, only the second term gives a contribution

$$
\overline{d N}_{r}=\frac{4}{15} \pi n g_{1}(r) r^{2} d r .
$$

Following this procedure in the simulation, $g_{1}(r)$ can be determined. The result can be used in the equation for shear viscosity Eq. (2.2). In fact the above procedure is a direct evaluation of the angular integrations in Eq. (2.2).

To overcome statistical noise in the pair distribution function, one has to average over all particles in the box and over a number of time steps. The number of time steps needed to reduce the (relative) statistical error in the perturbation of the pair distribution function rapidly increases with decreasing shear rate, because the amplitude $g_{1}(r)$ is proportional to the shear rate.

\section{B. Simulation details}

\section{Simulation box size}

Simulations were done in a cubic box of length $L$ with $N$ particles. For the majority of the simulations the number $N$ was chosen as $4 m^{3}$ where $m$ is an integer number, because there are four particles in the fcc unit cell in three dimensions. These particle numbers are compatible with an fcc structure. In a finite system different particle numbers may prohibit the formation of a long-range fcc structure. Effects of the choice particle number can expected to be significant at volume fractions where phase transitions play an important role. It can be expected that a Brownian dynamics simulation is somewhat less dependent on the system size than molecular dynamics simulations, because velocity correlations induced by the periodic boundaries are absent and only spatial correlations remain. Even with 32 particles reasonable results were obtained at most volume fractions, although the system in this case approximately consists of three particles in all directions. If the particles are in a random state $(\varphi<0.494)$, hardly any influence of the box size was found for $N \geqslant 108$. Nevertheless, most simulations were performed with 256 to 2048 particles. For $\varphi>0.49$ the influence of the box size will increase, because of the possibility of (partial) transitions towards states with long-range order.

Increasing the number of particles in the simulation reduces the statistical error in the pair distribution function of one time step, possibly allowing a smaller number of simulation time steps. However, increasing the number of particles reduces the error in the pair distribution function contact value only to a moderate extent: although the number of pairs to determine the pair distribution function increases quadratically with the number of particles $N$, the number of pairs at contact increases only linearly with $N$, because the number of neighbors at contact is limited to 12 at closest packing. So to reduce statistical error in the pair distribution function contact value, increasing the number of time steps is more efficient than increasing the number of particles, because of the more than linear increase in computation time with the number of particles.

\section{Simulation time}

A simulation run is characterized by the square root of the variance $\delta=\sqrt{2 \Delta \tau}$ of the Gaussian distribution from which the random displacements are taken. This is equivalent to fixing the time step $\Delta \tau$. The variance $\delta$ is the average 
squared displacement of a particle and must be chosen to be small compared to the particle diameter. Typical values for $\delta$ vary from $0.1 \sigma$ at very low volume fractions to $0.0075 \sigma$ at very high volume fractions. At high volume fraction small displacement steps are favorable, because the probabilities of an overlap of three or more particles (computationally expensive) and of an overlapping passage of two particles without collision detection, increase at high volume fractions. In most simulations we fixed the time step at $\Delta \tau=0.5$ $\times 10^{-4} \tau_{\sigma}$ by choosing $\delta=0.01 \sigma$, where $\sigma$ is the particle diameter. For comparison, after conversion of units this is five times smaller than the time step of Schaertl [45].

Equally important is the number of time steps in one simulation run. The number of time steps is both influenced by requirements of reducing the statistical error and by a wish to capture transient behavior.

The second important parameter is the shear rate of the flow, determined by the Péclet number Pe.

\section{Initial state}

The initial state is created for small volume fractions by random positioning of spheres, avoiding overlap configurations. At high volume fractions $(\varphi>0.25)$ this procedure does not yield an initial solution within reasonable time, and a different method must be used. A first possibility is to generate an fcc structure. This requires a number of relaxation time steps before the actual sampling is started, during which the fcc state melts or not. Because it is unknown how long the melting will take, and also how the completion of the melting should be measured, it is better to use the following method. Starting with a large simulation box and a small volume fraction, the simulation box is gradually decreased in size until the desired volume fraction is attained. The result is a random initial state. This procedure actually works in practice because after each decrease in size of the simulation box, the colloidal particles are allowed to move with the elastic collision correction method. This always generates a new, nonoverlapping configuration. Because the influence of the initial condition can be considerable for simulations at very high volume fractions in or near equilibrium, we used the second method to obtain an initially random initial state. Nevertheless, in our simulations we monitored changes in average values carefully to detect transient behavior. At almost all volume fractions and shear rates initially transient behavior was observed.

Summarizing we conclude that the simulation size influences both the finite-size errors and the statistical error in the pair distribution function. The simulation length influences both the statistical error and the possibility of detection of time-dependent behavior. Finally, the initial state influences the time dependent (physical) behavior, but in a way strongly affected by the simulation size. In practice, some balance has to be found between simulation size and simulation length.

A switch was built into the program to run a twodimensional version. The two-dimensional version was used to view a real-time simulation on screen to check the implementation of the periodic boundary conditions and the collision algorithm. Another advantage is that the simulations run faster, which is convenient in the development stage of the program. Some useful equations for a two-dimensional suspension of hard spheres are given in the Appendix. The simulations were done on the SGI Power Challenge computer with R10000 processors at the University of Twente.

\section{Equilibrium simulations}

In Fig. 1(a) we already plotted a pair distribution function obtained in an equilibrium simulation at zero shear rate. This can be compared with known results to check the simulation algorithm. Our simulation reproduces for instance the contact values of the pair distribution function as predicted by the Carnahan-Starling equation accurately. The equilibrium phase behavior of hard-sphere systems was already investigated with the Schaertl and Sillescu algorithm in Ref. [45]. Therefore, we will only quote some important results, before turning our attention to the nonequilibrium simulations and the shear viscosity.

In equilibrium the simulations should be equivalent to Monte-Carlo simulations of a hard-sphere gas. The maximum close packing fraction of hard spheres is $\varphi_{\mathrm{cp}}=0.74$, while the random close packing volume fraction is $\varphi_{\text {rcp }}$ $\approx 0.63$. In hard-sphere gas systems a freezing transition has been reported at volume fraction $\varphi=0.494 \pm 0.002$ by Hoover and Ree [46], and a melting transition of the crystalline hexagonal phase at $\varphi=0.545 \pm 0.002$. These results were confirmed by the computer simulations of Schaertl [45], aimed at establishing the phase behavior of colloidal suspensions for the same model as described in this paper.

These observations provide a setting for the nonequilibrium simulations. In the random fluid state, for $\varphi<0.494$, the zero steady shear viscosity exists and can be found from the simulations. In a solid state, by definition an object does not flow and resists small, steady deformations. The zero steady shear viscosity as we defined it does not exist. In any coexistence region between fluid and solid, part of the system is in a fluid state, and for large systems we expect the possibility of flowing. In a finite simulation box, however, a solid cluster may percolate over the simulation box and create a frozen state through the periodic boundary condition. Therefore, although a zero steady shear viscosity should exist in the coexistence region, it may be more difficult to find in the simulations as the volume fraction increases.

The occurrence of the coexistence region is still well below the highest volume fractions reported in the measurements of the steady shear viscosity of Van der Werff and De Kruif [3]. The experimental zero shear viscosity curve displays no strange behavior at the volume fractions of this coexistence region. An abrupt change in the characteristic stress, however, has been reported [47] at a volume fraction $\varphi=0.5$ and was associated with hard-sphere phase behavior. The characteristic stress is the stress at the shear rate $\dot{\gamma}_{c}$ for which $\eta\left(\dot{\gamma}_{c}\right)=\frac{1}{2}[\eta(0)+\eta(\infty)]$.

\section{Nonequilibrium simulations}

\section{Pair distribution function}

In nonequilibrium simulations there are no wellestablished results to which our results can be compared and checked. An important check is to reproduce the exact low shear, low-density perturbation of the pair distribution function, given by Eq. (2.7). The low-density solution was verified for both the three- and two-dimensional simulations. In 

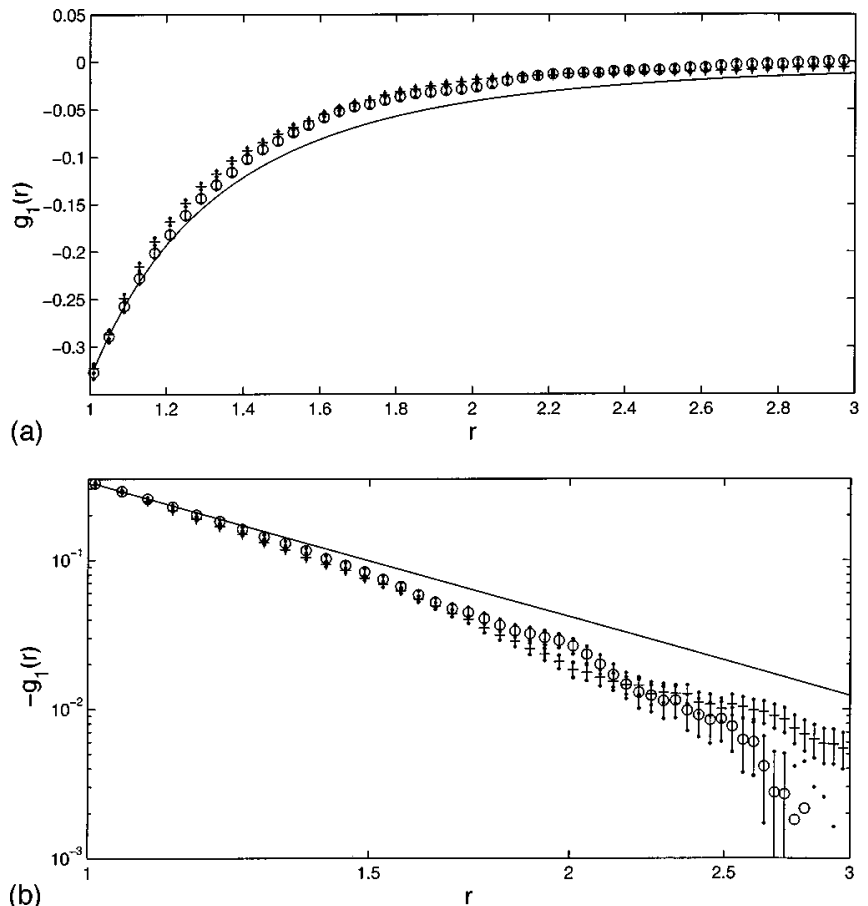

(b)

FIG. 2. Perturbation of the pair distribution function in steady shear flow $g_{1}(r)$ at a Péclet number $\mathrm{Pe}=0.5$ and for volume fractions $\varphi=0.01$ (open circles) and $\varphi=0.02$ (pluses), in a simulation of 108 particles. The total simulation time is $15000 \tau_{\sigma}$. Also, the low-density solution of Eq. (2.7) is plotted (solid line). See the text for a discussion of this plot. (a) Linear plot and (b) double logarithmic plot.

Fig. 2(a) the result is shown for a steady shear simulation at volume fractions $\varphi=0.01$ and $\varphi=0.02$, and a dimensionless shear rate of $\mathrm{Pe}=0.5$. The computational work required for these plots is considerable. The total simulation time was $\tau$ $=15000$, including a relaxation time of $\tau=7000$. This corresponds to $\sim 3 \times 10^{8}$ time steps with 108 particles and $\delta$ $=0.01$, taking about ten days on a single CPU of a SGI Power Challenge computer with $\mathrm{R} 10000$ processors. The statistical error is still large for $r \gtrsim 2$, and only in the range between $r=1$ and $r=2$ linearity in $\varphi$ is visible. This is more clear in the double logarithmic plot of $-g_{1}(r)$ in Fig. 2(b). However, in view of the statistical error, and of the development of the statistical error in time, we are confident that the theoretical low-density limit is approached eventually.

As a first example of the perturbation of the pair distribution function $g_{1}(r)$ at higher volume fractions, we determined $g(r)$ at a shear rate $\mathrm{Pe}=0.5$ and a volume fraction $\varphi=0.45$. At this volume fraction the particles are still in a random state. At these high volume fractions, $g_{1}(r)$ inherits much of the structure of the equilibrium pair distribution function $g_{0}(r)$, although the ratio $g_{1}(r) / g_{0}(r)$ is not a simple function. In Fig. 3 we have plotted the functions $g_{1}(r)$ and $g_{1}(r) / g_{0}(r)$. It can be seen that this perturbation function, calculated as discussed above, is rather smooth, in the sense that statistical fluctuations are sufficiently suppressed after $10^{5}$ time steps, corresponding to $5 \tau_{\sigma}$. This is somewhat surprising, because we are only considering small perturbations from equilibrium. Nevertheless, at lower shear rates the number of time steps required to sufficiently reduce statistical error increases rapidly.

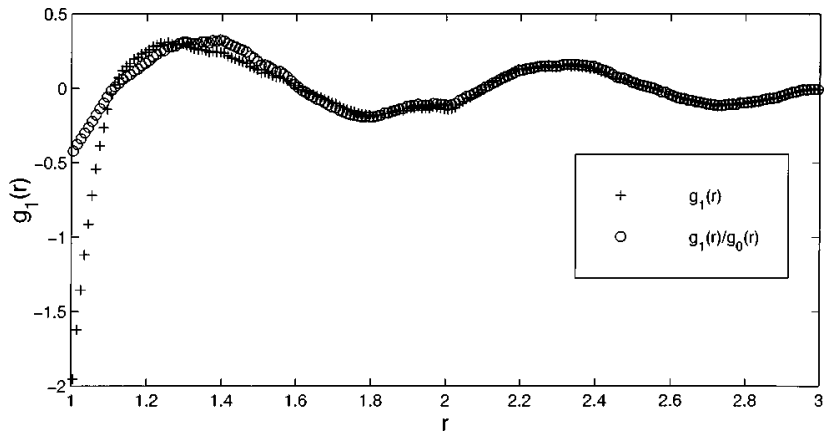

FIG. 3. Perturbation of the pair distribution function in steady shear flow, the functions $g_{1}(r)$, and the ratio $g_{1}(r) / g_{0}(r)$, for Péclet number $\mathrm{Pe}=0.5$ at a volume fraction $\varphi=0.45$, in a simulation of 512 particles.

A similar simulation run with the Schaertl and Sillescu overlap correction method predicted a contact value of $g_{1}$ different by $30 \%$. This confirms the expectations stated in Sec. III B that in nonequilibrium simulations the effect of the overlap correction methods is more pronounced than in equilibrium simulations.

\section{Shear viscosity}

In several steady shear simulations we have determined the contact value of the perturbation of the pair distribution function, and calculated the relative shear viscosity with Eq. (2.4) as a function of the Péclet number. The result is shown in a flow curve in Fig. 4, in which the shear viscosity is plotted as a function of shear rate. The contact values of the function $g_{1}(r)$ were determined up to a statistical error of

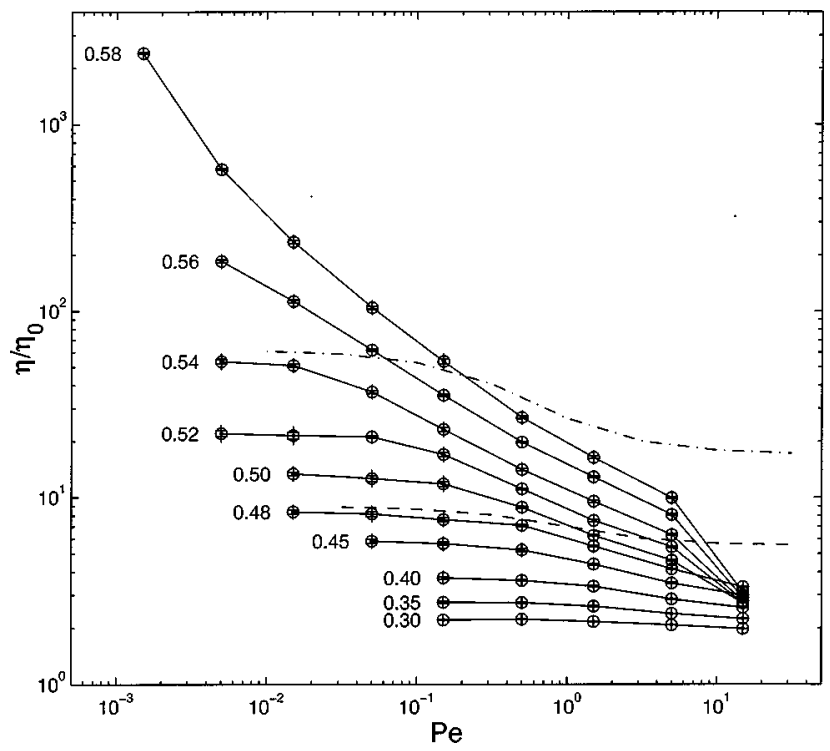

FIG. 4. Steady-state shear viscosity as a function of the Péclet number Pe for various volume fractions, in simulations of 1372 or 2048 particles. For comparison, two fit curves representing measurements of Van der Werff and De Kruif [3] are plotted for system SJ14 at $\varphi=0.419$ (dashed line) and $\varphi=0.538$ (dash-dotted line). At a shear rate of $\mathrm{Pe}=15.0$ the viscosity drops sharply at high volume fractions as the result of a transition to a state of hexagonally ordered strings with the strings in the direction of the flow. See also the text. 
$3 \%$. This error is also plotted, but is hardly discernible. The actual deviation of the viscosity from the model value is more related to finite-size effects and to transient or timedependent behavior of the system. For the results presented in Fig. 4 we tried to eliminate the latter errors as much as possible by increasing the simulation box as much as needed, and by monitoring transient behavior. If nontransient timedependent behavior was observed, the averaging interval was extended to capture an appropriate average value. We will discuss this shortly. For comparison, two fit curves representing measurements of Van der Werff and De Kruif [3] are plotted in Fig. 4 as dashed and dash-dotted lines. The experimental curves plotted correspond to the data for silica particles in cyclohexane (system SJ14) at volume fractions 0.419 and 0.538 .

(a) High shear rates and the shear thinning region. The first observation from Fig. 4 is that in general the results do not correspond to the measurements. This is mainly caused by the failure of the model to predict the shear viscosity at high Péclet numbers, which is dominated by hydrodynamic interactions. Therefore, in our simulations shear thinning behavior is more pronounced than in the measurements. If the behavior of the viscosity at high shear rates is disregarded or corrected, e.g., as in [5] with an empirical $\eta_{\infty}$, it is seen that the location of the shear thinning region is only roughly correct.

At a shear rate of $\mathrm{Pe}=15.0$ the viscosity drops sharply at high volume fractions as the result of a transition to a state of hexagonally ordered strings with strings in the direction of the flow. This has been observed by many authors, e.g., $[48,49]$ and has been the subject of extensive research for some time $[5,50,17]$. The lower volume fractions display similar transitions at higher shear rates. Transitions to states of long-range ordered strings were also observed at lower shear rates in smaller simulation boxes. However, such transitions could be repressed considerably by increasing the simulation box. This means that the long-range ordered states are at least to some extent an artifact of the finite simulation size, although they are certainly induced by the shearing motion.

Shear thickening as observed by several authors with simulation models including hydrodynamic interactions $[17,18]$ is not seen in our simulations. The shear induced ordering in strings in the direction of the flow seems a stable state in our model suspension, and breaking up of strings at high Péclet numbers was not observed. Although the shear thickening was not seen in the experiments of Van der Werff and De Kruif, it has been established in more recent experiments $[15,16]$.

Apparently the model without hydrodynamic interactions lacks essential interaction mechanisms at high Péclet numbers, and results for this model should be interpreted with care in this regime. The regime at high rates, however, is not the main goal of this paper for a different reason. Although the simulation algorithm is stable up to very high shear rates if the time step is accordingly decreased, the validity of the superposition of diffusion and velocity field (Sec. III A) is only rigorous at small shear rates.

(b) Low shear region. In this paper we are particularly interested in the low shear viscosity, because it is expected that in the low shear regime, where Brownian motion is

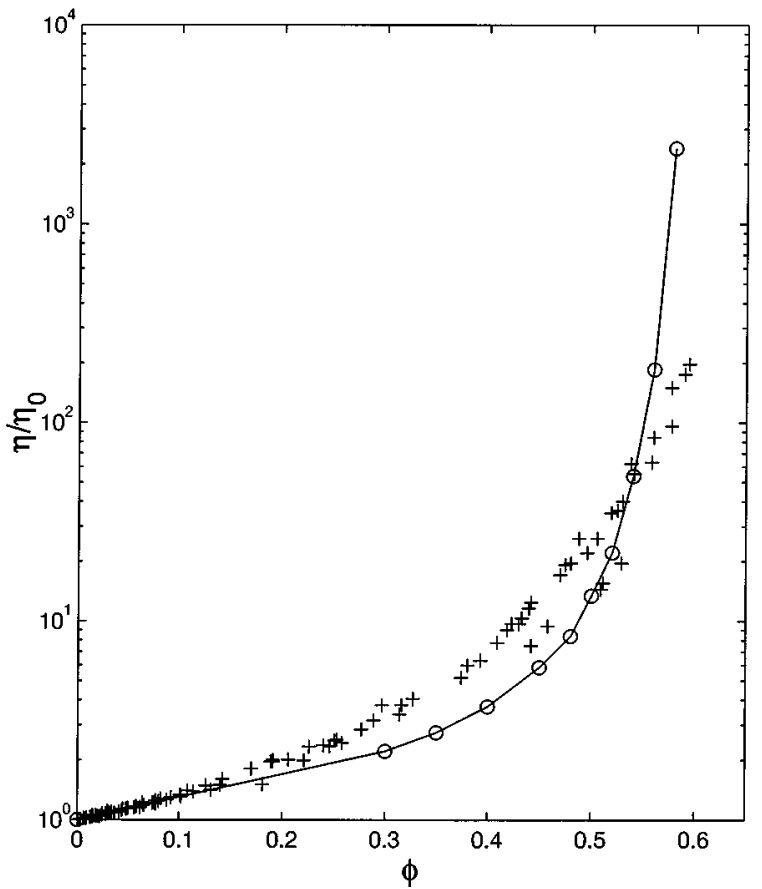

FIG. 5. Relative shear viscosity as a function of the volume fraction $\varphi$ from Brownian dynamics computer simulations in the limit of zero shear rate (open circles), in comparison to the measurements $(+)$ on four different systems of silica particles in cyclohexane by Van der Werff and De Kruif [3]. Note that for $\varphi>0.54$ the value is not the plateau value.

dominant, the suspension model performs best. Due to the computational efficiency of the Brownian dynamics algorithm, allowing many particles and many time steps, viscosities can be "measured" from the simulation at quite low Péclet numbers.

On the Newtonian plateau the viscosity is constant as a function of the shear rate and the perturbation amplitude $g_{1}$ is linear in the shear rate. In Fig. 4 it is seen that the Newtonian plateau has been attained for volume fractions up to $\varphi=0.54$. We have plotted the shear viscosity at the lowest shear rate simulated, as a function of volume fraction in Fig. 5 . The experimental data on the zero shear viscosity for all four experimental systems of Van der Werff and De Kruif [3] are plotted for comparison. We did not use a fitting procedure as suggested by these authors to obtain a zero shear viscosity for $\varphi>0.54$, because it is not a priori clear that the shape of the flow curve is preserved across the hard-sphere phase transition region.

Figure 5 shows that, as compared to the experiments, the viscosities obtained from the computer simulations fail at intermediate and low volume fractions. Only at high volume fractions does the model viscosity rapidly catch up with the experimental viscosity.

These results suggest that at low volume fractions hydrodynamic effects give the most important contribution to the shear viscosity in a real suspension, which can be expected because the $\varphi^{2}$ term in the expression for the viscosity is incorrect when compared to the $\varphi^{2}$ result for the zero shear viscosity of Batchelor and Green [28,2],

$$
\frac{\eta}{\eta_{0}}=1+\frac{5}{2} \varphi+6.2 \varphi^{2}
$$




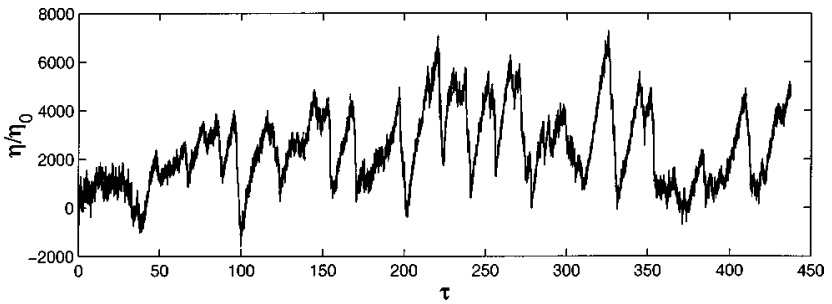

FIG. 6. Relative shear viscosity as a function of dimensionless time at a Péclet number $\mathrm{Pe}=0.0015$ and a volume fraction $\varphi$ $=0.58$, in a simulation of 2048 particles. After $40 \tau_{\sigma}\left(8 \times 10^{5}\right)$ the system transfigures from a mostly random state towards a state with regions of local order. No "final" state is corroborated for the duration of this simulation.

Moreover, because the model viscosity is even larger than the experimental viscosity for $\varphi>0.54$, the results suggest that at high volume fractions the hydrodynamic interactions prevent the hard-sphere interactions from becoming too dominant. However, this is somewhat less conclusive, because the errors in the measurements of volume fractions or deviations from monodispersity both have large effects on the viscosity at volume fractions close to the close packing volume fraction $\varphi_{\text {rcp }} \approx 0.63$.

Also in the low shear region phase transitions are responsible for transient behavior. For volume fractions beyond the start of the phase coexistence region $\varphi>0.494$ transitions to states of partially crystalline structure were observed. In contrast to the high shear transitions described above, the system does not evolve towards a (meta)stable steady state of hexagonally layered strings at small shear rates, but rather to a collection of regions of hexagonal packing. In Fig. 6 the history of the relative shear viscosity is plotted for $\varphi=0.58$ and $\mathrm{Pe}=0.0015$. After being in a random, fluidlike state for about $40 \tau_{\sigma}$, the system tranfigures rather sharply into a state with ordered regions. A typical example of these regions is shown in Fig. 7.

A single viscosity can be ascribed to such systems only if the averaging time interval is long enough, because the fluctuations in Fig. 6 occur on a much longer time scale than the fluctuations in the purely random state. Correct treatment of these states requires either a strong increase in simulation length or in simulation size. Moreover, because ordered re- gions are easily of the same size as the simulation box, an additional, lower limit on the simulation size is set by the demand to capture these regions entirely. As an example, we feel that this requirement has been (scarcely) met in Fig. 7 in a simulation of 2048 particles. Domainlike states can be expected in the coexistence region, but in a similar simulation at zero shear rate, a transition occurred at a later time $\left(80 \tau_{\sigma}\right)$. Also, the simulation box displayed almost global order. A small shearing motion apparently accelerates structural relaxation, and decreases the average domain size.

It will be interesting to study the relations between the fluctuations in the shear viscosity, the sizes and shapes of the ordered regions, and the shear rate. This is subject of current research. It is in this field that the present suspension model may be a powerful tool, even though the results in this paper demonstrate the inadequacy of the hard-sphere suspension model without hydrodynamic interactions to describe the high shear regime of real suspensions.

\section{CONCLUSIONS}

In this paper we have discussed a model of hard-sphere suspensions in which hydrodynamic interactions are neglected, but single-particle hydrodynamics and hard-sphere interactions are retained. The aim was to treat this model consistently, and with no additional approximations, to establish its merits in describing the rheology of real suspensions.

After discussing an expression for the shear viscosity, we described a simulation algorithm that is both accurate and efficient to simulate the model suspension. This simulation algorithm is consistent with the expression for the viscosity. In addition, the algorithm was compared with two alternative, similar algorithms, and was shown to be better suited for the purpose of determining the excluded volume effects in a suspension dominated by Brownian motion. In equilibrium simulations the algorithm is able to reproduce the hardsphere pair distribution function accurately, and in nonequilibrium simulations the exact low-shear, low-density limit of the perturbation of the pair distribution function could be computed.

The algorithm was used to study the steady shear viscosity. The validity of the model of the hard-sphere suspension
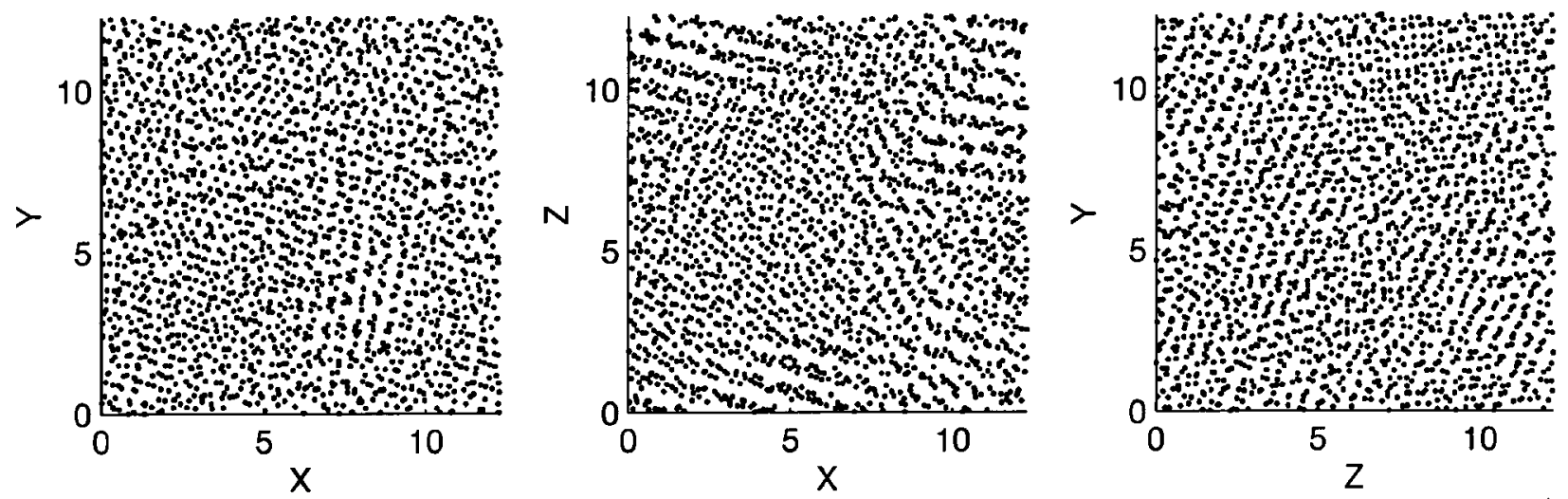

FIG. 7. Typical snapshot of the simulation box of a simulation of 2048 particles at a Péclet number $\mathrm{Pe}=0.0015$ for projection on the three Cartesian planes. The flow is in the $X$ direction and the velocity gradient is in the $Y$ direction. The volume fraction is $\varphi=0.58$. For transparency, the particles are not drawn at their actual size. This snapshot was taken at $\tau \approx 200$; see also Fig. 6 . 
was tested through comparison with the experimental work of Van der Werff and De Kruif. In general, it is concluded that the results of the hard-sphere model without hydrodynamic interactions is disappointing.

In the nonequilibrium simulations we found that in the intermediate and high shear regimes the model heavily underestimates the shear viscosity of real suspensions. The shear thinning region is found only roughly at the correct position. We have found shear induced ordering in strings at high shear rates as usual, but have not encountered shear thickening. It is concluded that hydrodynamic interactions indeed are non-negligible in these regimes at all volume fractions.

In the low shear limit we found that the zero shear viscosity is underestimated at low and moderate densities, as compared to measurements of a real suspension, but the viscosity is overestimated as compared to the experimental values at high densities in the phase transition regime. There is a possibility that the experimental systems fail at extremely high volume fractions, as they do not consist of truly hard, monodisperse spheres, and that the measured shear viscosity is inaccurate. A more likely explanation is that the hydrodynamic interactions help the colloidal particle to find an easier way through the sample. This would be, again, a fundamental hydrodynamic property of the suspension.

A problem when determining the zero shear viscosity is that at high volume fractions the Newtonian plateau is increasingly difficult to find. The simulation times increase rapidly with decreasing shear rate in order to overcome statistical noise. Furthermore, the simulation box must be chosen large to avoid finite size errors in the phase transition regime. It was found that at high volume fractions the behavior is complex, with a strong interplay of box size, shear rate, and spontaneous or shear induced phase transitions. The existence of a zero steady shear viscosity has been established in our simulations for volume fractions $\varphi$ up to 0.54 .

The complete phase behavior of the hard-sphere system in the region for $\varphi>0.494$, in relation to the shear rate and the simulation box size, requires more attention than is possible in this paper. This is a subject of current research. More simulations, with even more particles and perhaps even smaller shear rates, may be required to explore this area of the shear viscosity satisfactorily. In this field computationally efficient models such as the one used in this paper may still provide valuable results that are relevant for real suspensions, although quantitatively correct results should not be expected.

\section{APPENDIX: TWO-DIMENSIONAL SUSPENSIONS}

In two dimensions the volume fraction is $\varphi=\frac{1}{4} \pi \sigma^{2} N / V$ for a system of hard disks of diameter $\sigma$. The hexagonal close packing volume fraction is $\varphi_{\text {rcp }}=\pi / 2 \sqrt{3}=0.907$.

In a two-dimensional system $[\mathbf{r}=(x, y)$ and $r$ $\left.=\sqrt{x^{2}+y^{2}}\right]$, the Smoluchowski equation for the low-density pair distribution function is Eq. (2.5). In a weak flow expansion, the pair distribution function will have the form

$$
\psi(\mathbf{r})=\psi_{0}(r)+2 \mathrm{Pe} \cos \phi \sin \phi \psi_{1}(r) .
$$

The two-particle Smoluchowski equation simplifies to

$$
\psi_{1}^{\prime \prime}+\frac{1}{r} \psi_{1}^{\prime}-\frac{4}{r^{2}} \psi_{1}=0
$$

and $\psi_{1}^{\prime}(1)=1$. The solution of this equation is

$$
\psi_{1}(r)=-\frac{1}{2 r^{2}}
$$

which predicts a nonequilibrium low shear, low-density contact value of $-\frac{1}{2}$. In three dimensions this was $-\frac{1}{3}$. The equations corresponding to the three-dimensional equations (4.1) and (4.4) are

$$
d N_{r}=n \psi_{0}(r) 2 \pi r^{2} d r
$$

and

$$
\overline{d N}_{r}=\frac{1}{4} \pi n \psi_{1}(r) r d r .
$$

[1] G. K. Batchelor, J. Fluid Mech. 41, 545 (1970).

[2] G. K. Batchelor, J. Fluid Mech. 83, 97 (1977).

[3] J. C. van der Werff and C. G. de Kruif, J. Rheol. 33, 421 (1989).

[4] B. J. Ackerson and P. N. Pusey, Phys. Rev. Lett. 61, 1033 (1988).

[5] D. M. Heyes and J. R. Melrose, J. Non-Newtonian Fluid Mech. 46, 1 (1993).

[6] J. A. Leegwater and G. Szamel, Phys. Rev. A 46, 4999 (1992).

[7] M. Medina-Noyola, Phys. Rev. Lett. 60, 2705 (1988).

[8] E. G. D. Cohen and I. M. de Schepper, J. Stat. Phys. 63, 241 (1991).

[9] P. Strating, Ph.D. thesis, University of Twente, Enschede, The Netherlands, 1995.

[10] I. M. de Schepper, H. E. Smorenburg, and E. G. D. Cohen, Phys. Rev. Lett. 70, 2178 (1993).
[11] J. F. Brady, J. Chem. Phys. 99, 567 (1993).

[12] P. Strating, J. Chem. Phys. 103, 10226 (1995).

[13] N. J. Wagner and W. B. Russel, Physica A 155, 475 (1989).

[14] R. A. Lionberger and W. B. Russel, J. Chem. Phys. 106, 402 (1997).

[15] J. Bender and N. J. Wagner, J. Rheol. 40, 899 (1996).

[16] P. D'Haene, J. Mewis, and G. G. Fuller, J. Colloid Interface Sci. 156, 350 (1993).

[17] T. N. Phung and J. F. Brady, J. Fluid Mech. 313, 181 (1996).

[18] R. C. Ball and J. R. Melrose, Physica A 247, 444 (1997).

[19] J. R. Melrose, J. H. van Vliet, and R. C. Ball, Phys. Rev. Lett. 77, 4660 (1996).

[20] M. Doi and S. F. Edwards, The Theory of Polymer Dynamics (Oxford University Press, New York, 1992).

[21] R. J. J. Jongschaap and J. Mellema, J. Rheol. 39, 953 (1995).

[22] A. Einstein, Ann. Phys. (Leipzig) 19, 289 (1906). 
[23] A. Einstein, Ann. Phys. (Leipzig) 34, 591 (1911).

[24] L. D. Landau and E. M. Lifshitz, Fluid Mechanics (Pergamon, London, 1959).

[25] J. P. Hansen and I. R. McDonald, Theory of Simple Liquids, 2nd ed. (Academic, London, 1990).

[26] W. B. Russel, D. A. Saville, and W. R. Schowalter, Colloidal Dispersions (Cambridge University Press, Cambridge, 1989).

[27] A. C. Eringen, Mechanics of Continua, 2nd ed. (Krieger, Malabar, FL, 1980).

[28] G. K. Batchelor and J. T. Green, J. Fluid Mech. 56, 401 (1972).

[29] J. D. Jackson, Classical Electrodynamics, 2nd ed. (Wiley, New York, 1975).

[30] B. J. Alder and T. E. Wainwright, J. Chem. Phys. 27, 1208 (1957)

[31] J. M. V. A. Koelman and P. J. Hoogerbrugge, Europhys. Lett. 21, 363 (1993).

[32] D. L. Ermak and J. A. McCammon, J. Chem. Phys. 69, 1352 (1978).

[33] G. Bossis and J. F. Brady, J. Chem. Phys. 80, 5141 (1984).

[34] E. M. Toose, Ph.D. thesis, University of Twente, Enschede, The Netherlands, 1997.

[35] N. G. van Kampen, Stochastic Processes in Physics and
Chemistry (North-Holland, Amsterdam, 1992).

[36] D. M. Heyes and A. C. Brańka, Phys. Rev. E 50, 2377 (1994).

[37] D. M. Heyes, P. J. Mitchel, and P. B. Visscher, J. Chem. Soc., Faraday Trans. 90, 1133 (1994).

[38] J. M. van der Veer, Ph.D. thesis, University of Twente, Enschede, The Netherlands, 1992 (unpublished).

[39] D. M. Heyes, J. Non-Newtonian Fluid Mech. 27, 47 (1988).

[40] D. M. Heyes, Phys. Lett. A 123, 399 (1988).

[41] B. Cichocki and K. Hinsen, Physica A 166, 473 (1990).

[42] W. Schaertl and H. Sillescu, J. Stat. Phys. 74, 678 (1994).

[43] D. J. Evans and G. P. Morriss, Statistical Mechanics of Nonequilibrium Liquids (Academic, London, 1990).

[44] M. P. Allen and D. J. Tildesley, Computer Simulation of Liquids (Clarendon, Oxford, 1990).

[45] W. Schaertl, J. Stat. Phys. 79, 299 (1995).

[46] W. G. Hoover and F. H. Ree, J. Chem. Phys. 49, 3609 (1968).

[47] C. G. de Kruif, E. M. F. van Iersel, A. Vrij, and W. B. Russel, J. Chem. Phys. 83, 4717 (1985).

[48] L. V. Woodcock, Phys. Rev. Lett. 54, 1513 (1985).

[49] D. M. Heyes, G. P. Moriss, and D. J. Evans, J. Chem. Phys. 84, 4760 (1985).

[50] W. H. Boersma, J. Rheol. 39, 841 (1995). 